\title{
Endoscopic Tympanoplasty: Is it Better than Microscopic Tympanoplasty? Our Experience
}

\author{
Tarun Ojha', Vipasha Yogi², Anuj Kansara ${ }^{3}$, Kunal Sharma ${ }^{4}$
}

\begin{abstract}
Objective: To evaluate the efficacy and compare the results of conventional tympanoplasty with endoscopic tympanoplasty. Materials and methods: In this study, patients with clinically diagnosed chronic suppurative otitis media with dry central perforation were included. A total of 200 cases were selected of which 100 cases underwent conventional microscopic tympanoplasty and 100 cases underwent endoscopic tympanoplasty. Follow-up of all patients were done on postoperative day 7, 30, and third month of surgery. Comparison of postoperative complications, graft uptake, and average gain in hearing was done.

Results: Success rate of graft uptake was $96 \%$ (96/100) in conventional microscopic group of tympanoplasty, 92\% (92/100) in endoscopic group of tympanoplasty with average hearing gain of $13.96 \mathrm{~dB}$ in conventional group and $15.03 \mathrm{~dB}$ in endoscopic group.

Conclusion: The surgical outcome of endoscope-assisted tympanoplasty was comparable to the conventional microscope-assisted tympanoplasty in terms of graft uptake and hearing improvement. In endoscopic tympanoplasty group, patients had better results in terms of cosmesis and postoperative recovery. Endoscopic tympanoplasty approach can be used as a good alternative.

Keywords: Chronic otitis media, Conventional microscopic tympanoplasty, Endoscopic tympanoplasty, Tympanoplasty.

Journal of Mahatma Gandhi University of Medical Sciences \& Technology (2019): 10.5005/jp-journals-10057-0104
\end{abstract}

\section{INTRODUCTION}

Surgical procedures that are used for repair of the tympanic membrane and middle ear, respectively, are myringoplasty and tympanoplasty. ${ }^{1}$ Any operation involving reconstruction of the tympanic membrane with or without ossicular chain reconstruction is referred to as tympanoplasty. On the other hand, myringoplasty refers to repair of tympanic membrane only. The most widely used and accepted method is underlay graft technique of temporalis fascia or sometimes perichondrium. ${ }^{2}$ The introduction of the operating microscope has enhanced the outcome of myringoplasty by improving the accuracy of the technique.

With the introduction of the endoscope into other branches of surgery, there have been attempts at its utilization in otology. The first published description of imaging of the middle ear by endoscopy was by Mer et al. in 1967.

Endoscopic ear surgery (EES) has remained controversial since its first description in the English literature in the late 1960 s. $^{3}$ Modern microscopes provide excellent views of the surgical field and confer the ability for binocular vision and two-handed surgery, and visualization of deeper recesses in the middle ear is limited. The optical properties of a microscope require an adequate amount of light to reach the surgical plane. As a result, current microscopebased operative approaches frequently necessitate soft tissue retraction and/or bony drilling to adequately visualize the targeted pathology.

In contrast, endoscopes allow for improved visualization, as the light source is located at the distal tip of the instrument, and angled lenses offer a wide perspective of the operative field. Further, transcanal endoscopic approaches transform the external auditory canal into a surgical portal. Due to the relative diameter of the endoscope to the ear canal, however, only one-handed surgery is feasible, thereby making dissection less efficient and more challenging, especially in the case of blood in the operative field. ${ }^{4}$
${ }^{1}$ Head of Department and Professor, Department of ENT and Head \& Neck Surgery, Mahatma Gandhi Medical College and Hospital, Jaipur, Rajasthan, India

${ }^{2-4}$ Junior Resident ENT and Head \& Neck Surgery, Mahatma Gandhi Medical College and Hospital, Jaipur, Rajasthan, India

Corresponding Author: Tarun Ojha, Department of ENT and Head \& Neck Surgery, Mahatma Gandhi Medical College and Hospital, Jaipur, Rajasthan, India, Phone: +919414140711, e-mail: vipashadryo91@ gmail.com

How to cite this article: Ojha T, Yogi V, Kansara A, et al. Endoscopic Tympanoplasty: Is it Better than Microscopic Tympanoplasty? Our Experience. J Mahatma Gandhi Univ Med Sci Tech 2019;4(2):53-56.

Source of support: Nil

Conflict of interest: None

Further, questions remain as to the long-term safety of extended static application of endoscopes in the middle ear due to the heat generated by these instruments. ${ }^{5,6}$

The use of a rigid endoscope for myringoplasty has a significant advantage, as it is simple to use, not only for the examination but also for the repair of the tympanic membrane perforation. This provides a magnified vision and hence enables the surgeon to change rapidly from a closeup to a wide-angle view, just by going closer or by withdrawing the scope. Further, it provides an allround vision to the surgeon who can rotate the angled endoscope to visualize the deep anterior canal wall, anterior recess, anterior marginal perforations, sinus tympani, facial recess, hypotympanum, and the attic.

\section{Materials and Methods}

This study was carried out at Mahatma Gandhi Hospital, Jaipur, Rajasthan, from September 2018 to May 2019. In ENT OPD, patients who presented with complain of ear discharge and those who were 
willing for surgery with their informed and written consent were screened. 256-, 512-, and 1024-Hz frequency tuning forks were used for Tuning fork test, and pure tone audiometry was done to evaluate the type and degree of hearing loss. Hearing loss was calculated by taking the average of A-B gap at frequencies of 500,1 , and $2 \mathrm{kHz}$.

\section{Inclusion Criteria}

- Dry nondischarging ear

- Age-group 16-50 years

\section{Exclusion Criteria}

- Discharging wet ear

- Revision cases of tympanoplasty

- Cholesteatoma

- SNHL

- Tympanosclerosis

- Age less than 16 years

In all, 100 cases underwent endoscopic tympanoplasty and 100 cases underwent conventional microscopic tympanoplasty. Type 1 tympanoplasty was done in all cases. In all microscopic tympanoplasty procedures, temporalis fascia was used as graft material, while either TF or tragal perichondrium was used for endoscopic tympanoplasty as graft material. All microscopic tympanoplasty were operated by postaural route.

\section{Technique}

Zero-degree and $30^{\circ}$ endoscopes were used (Fig. 1). Direct visualization of all endoscopic surgeries were displayed on the monitor. Local anesthesia was used in all cases. In all cases of microscopic tympanoplasty procedure, temporalis fascia was used as graft material while either TF or tragal perichondrium was used as graft material in cases endoscopic tympanoplasty. All endoscopeassisted tympanoplasty was done through transcanal route. After harvesting temporalis fascia graft margins, the perforation was freshened using a wide curved pick. The middle ear inspection was done, and findings were noted: ossicular chain continuity and incudostapedial joints, patency of eustachian tube, and oval and the round window. Round window reflex visualized. Ossicular chain was intact. Underlay technique for graft was used in all the cases. Medicated gelfoam was used for stabilizing and packing over the

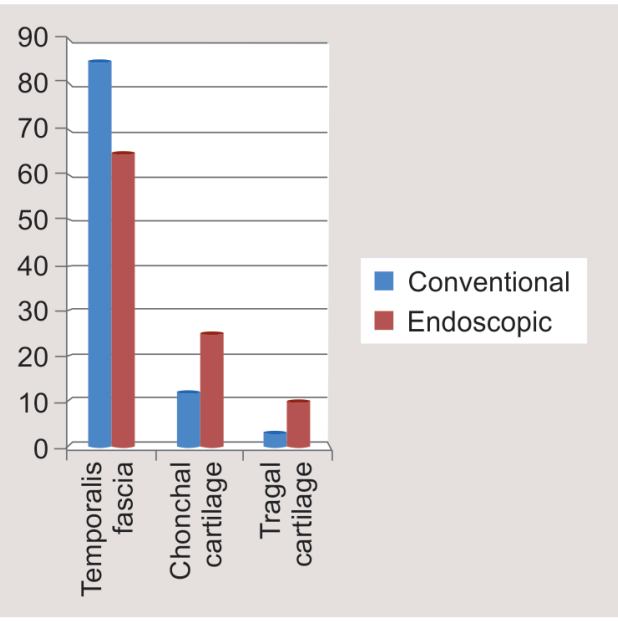

Fig. 1: Graft material graft. Postoperative antibiotics were given. The patients of both groups have been followed up on postoperative day 7 after one month and three months, and the immediate as well as delayed results in terms of efficacy, benefit, and postoperative complication were compared. In conservative tympanoplasty, suture removal was done on postoperative day 7. All cases were evaluated for postoperative pain, wound healing (any dehiscence or infection), and discharge on follow-up visits. At 3-month final assessment, graft uptake was done and postoperative PTA hearing was assessed.

\section{Pics Step Wise Both}

PIC Unit Endoscopy Unit

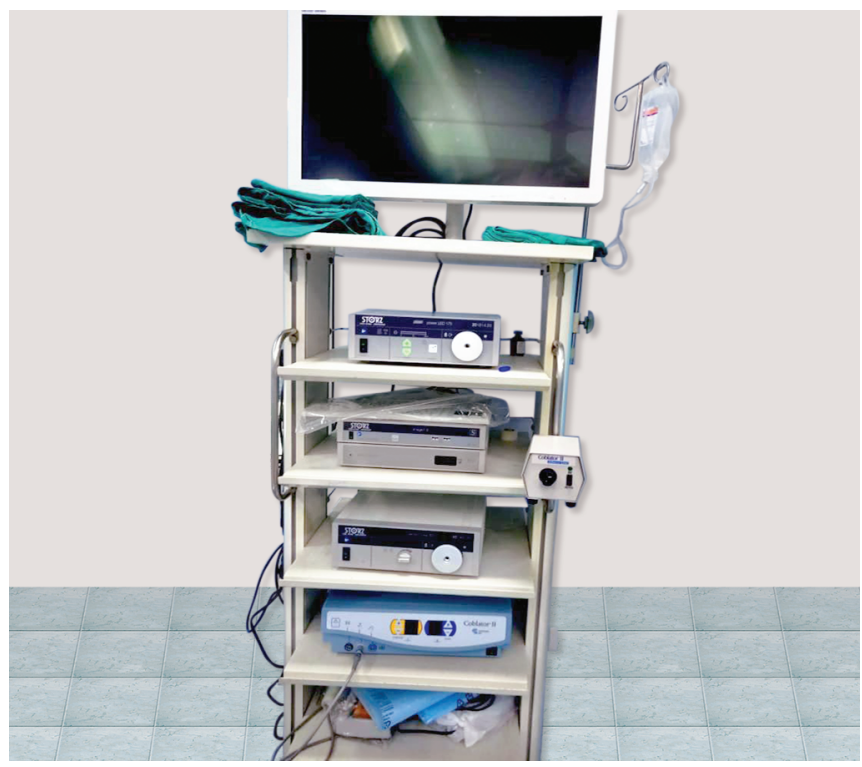

\section{PIC scope OTO ENDOSCOPE}

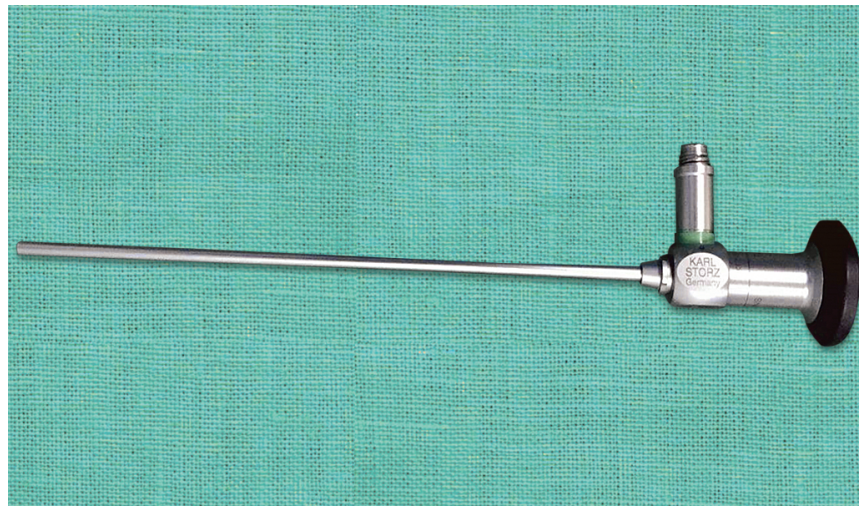

\section{ResUlts}

In this study of 200 cases of central tympanic membrane perforation, successful graft uptake after 3 months in conventional microscopic and endoscopic myringoplasty group was $96 \%$ and $92 \%$, respectively (Fig. 2). Average time taken in conventional microscopic tympanoplasty and endoscopic tympanoplasty group was in the 40-minute range (40-50 minutes) and 55-minute range (51-60 minutes), respectively (Fig. 3). Canaloplasty was performed in 12 of 100 patients in the microscopic group as required while in the endoscopic group, canaloplasty was not required in any 


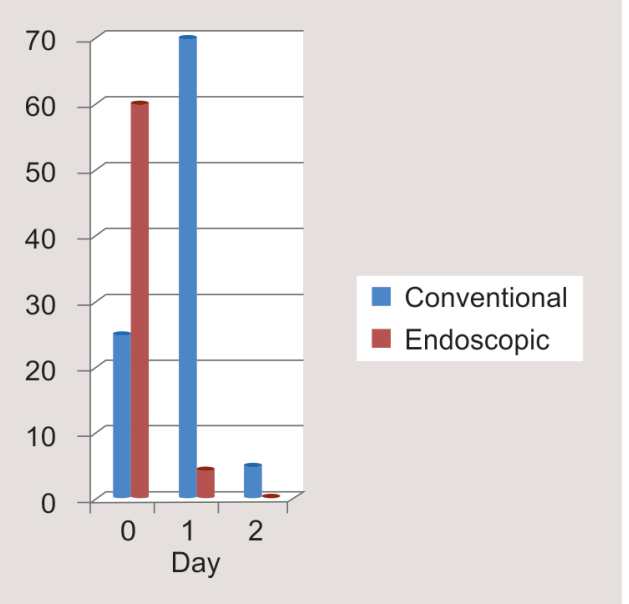

Fig. 2: Successful graft uptake

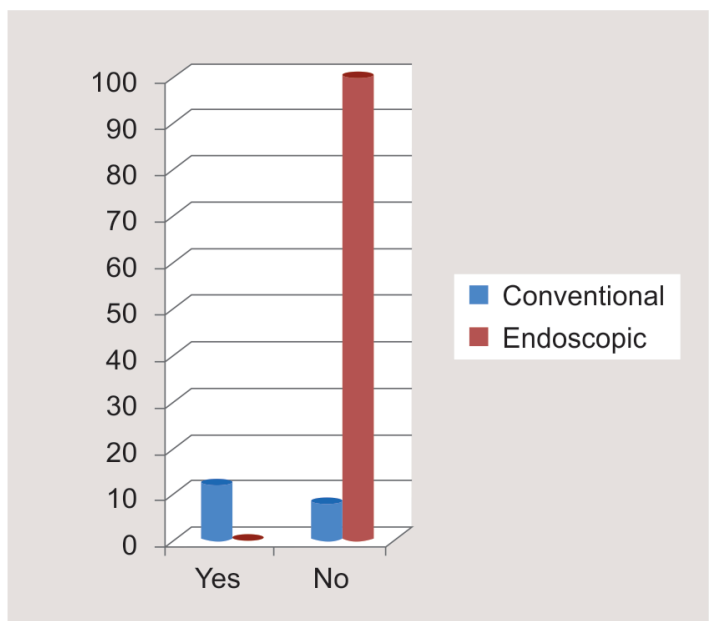

Fig. 4: Patient undergoes canaloplasty

case (Fig. 4). In our study, average preoperative hearing loss in conventional myringoplasty group was $31.53 \mathrm{~dB}$ while in endoscopic myringoplasty group it was $30 \mathrm{~dB}$. Postoperatively, average air-bone gap in conventional myringoplasty group was $16.03 \mathrm{~dB}$, while in endoscopic myringoplasty group it was $15 \mathrm{~dB}$. Average hearing gain in conventional myringoplasty group is $13.96 \mathrm{~dB}$ and in endoscopic myringoplasty group is $15.03 \mathrm{~dB}$ (Fig. 5). In all, 95\% of patients in the endoscopic tympanoplasty group rated their cosmetic result as excellent, whereas in the conventional group, $75 \%$ patient rated their cosmetic result as excellent, $22 \%$ patients rated their cosmetic result as satisfactory, and $3 \%$ patients rated their cosmetic result as poor (Fig. 6). So, endoscopic group of patients had better cosmetic result compared to conventional myringoplasty groups (Fig. 7). Average postoperative hospital stay in microscopic myringoplasty group was 1 day, while in endoscopic myringoplasty group maximum patients were discharged on the same day (Fig. 8). Patients present with postoperative complication such as wound gap in 4 of 100 in microscopic group while none in the endoscopic group (Fig. 6).

\section{Discussion}

The main aim of this study was to evaluate the advantage of using endoscopes in ear surgeries over conventional methods of ear

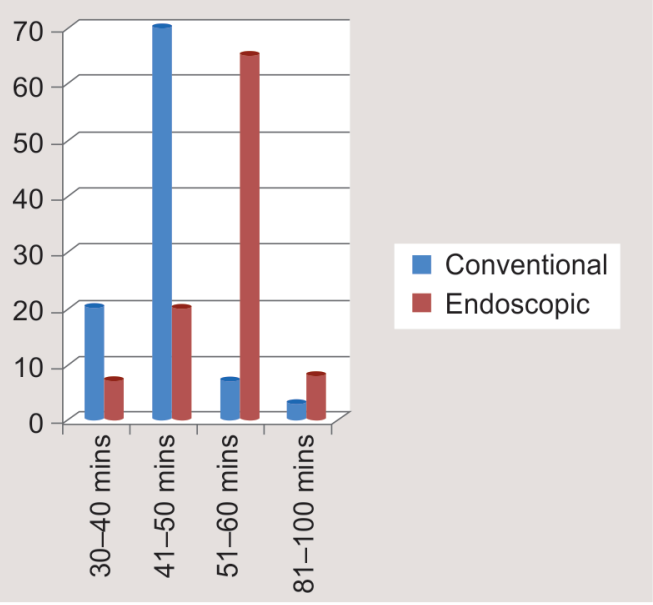

Fig. 3: Time taken

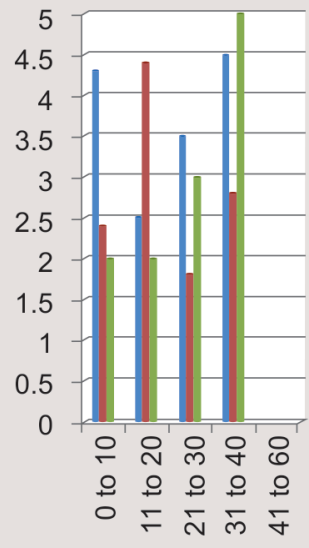

- Microscopic pre-op

- Microscopic post-op

Endoscopic pre-op

- Endoscopic post op

응 웅ㅇㅇ 8

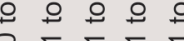

Fig. 5: Hearing assessment

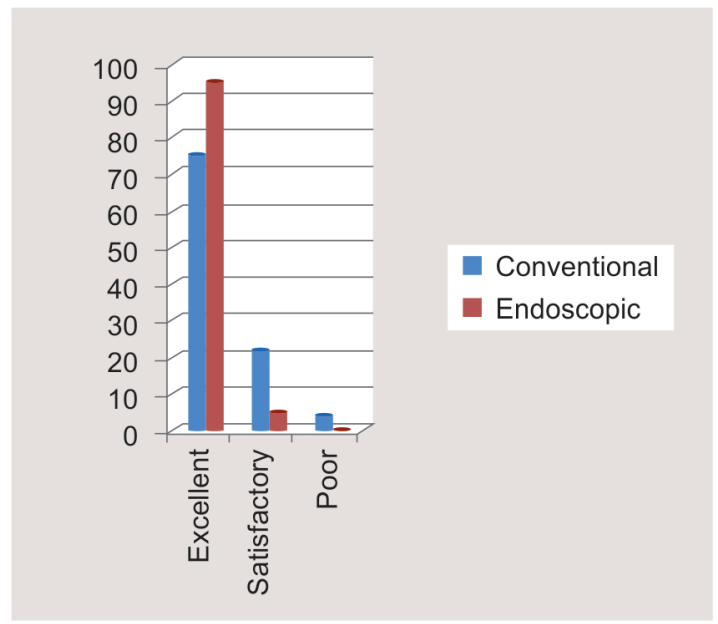

Fig. 6: Subjective cosmetic results

surgeries. In conventional tympanoplasty, group time taken was less compared to endoscopic group. As endoscopic surgeries are one-handed surgeries, due to bleeding, achieving hemostasis can be troublesome with endoscopes. In the conventional 


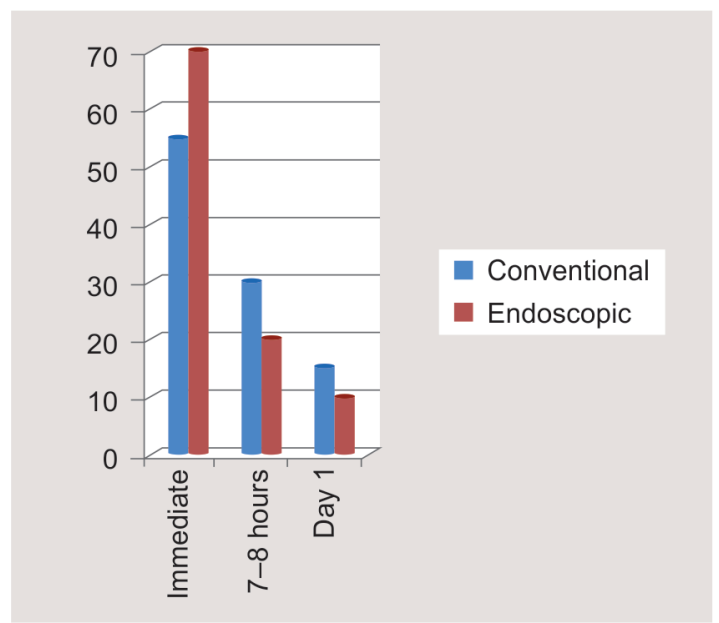

Fig. 7: Postoperative pain

group, feasibility of using instruments in both hands make it less troublesome. But this can be overcome with expertise of the surgeon. Endoscopes provide a wide and clean surgical field. In endoscopic surgeries, precise canal incision is given. Therefore, manipulation of soft tissue is minimal. As view is magnified and wide bone drilling or canaloplasty is not required, bleeding is less, which subsequently increased effectiveness of this method of surgery as well as reduced postoperative pain and complication, such as infection, delayed wound healing, and scar dehiscence. External incisions, soft tissue dissections, and mastoidectomies can be avoided by using endoscopes in selected cases. Microscopes give limited visualization of deep and hidden spaces involving sinus tympani, epitympanum facial recess, and the attic area. ${ }^{4,8-10}$ So, in microscopic techniques, further soft tissue dissection/retraction and bony drilling to obtain a better surgical view are frequently needed. ${ }^{4}$ In any patient of ET group, EAC widening or external incision was not needed. Otherwise, of patients who underwent conventional microscopic tympanoplasty, one-third patients had EAC widening by drilling. Endoscopic ear surgery has several disadvantages over conventional microscopic technique, such as the endoscopic instrument can cause direct injury and thermal damage to the external canal and middle ear. ${ }^{5,6}$ Due to heat generation from light source of the endoscope, Kozin et al. ${ }^{6}$ recommended using sub-maximal light intensity and frequent repositioning of the endoscope. No such complications were seen in this study group.

\section{Conclusion}

The surgical outcome of endoscopic tympanoplasty in terms of successful graft uptake and hearing gain was comparable to the conventional microscope-assisted tympanoplasty. In endoscopic

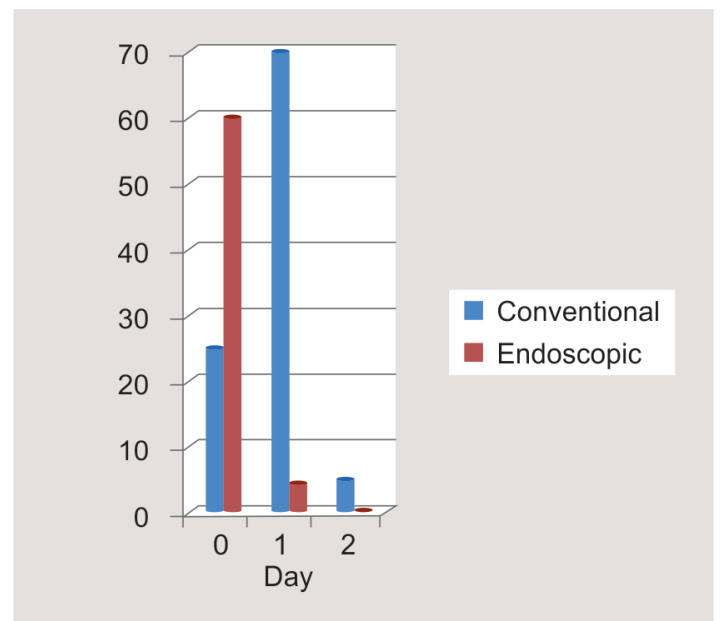

Fig. 8: Postoperative stay

tympanoplasty group, patients had better results in terms of cosmesis and postoperative recovery.

\section{References}

1. Patel J, Aiyer RG, Gajjar Y, et al. Endoscopic tympanoplasty vs microscopic tympanoplasty in tubotympanic csom: a comparative study of 44 cases. 2015;3(8):1953-1957. DOI: 10.18203/2320-6012. ijrms20150307.

2. Kumar M, Kanaujia SK, Singh A. A comparative study of endoscopic myringoplasty vs conventional myringoplasty. 2015;7(3):132-137. DOI: 10.5005/jp-journals-10003-1209.

3. Mer S, Derbyshire J, Brushenko A, et al. Fiberoptic Endotoscope for Examining the Middle Ear. Arch Otolaryngol 1967;85(4):61-67. DOI: 10.1001/archotol.1967.00760040389009.

4. Kozin ED, Gulati S, Lehmann A, et al. Systematic review of endoscopic middle ear surgery outcomes. Laryngoscope 2015;125(5):1205-1214. DOI: 10.1002/lary.25048.

5. Bottrill I, Perrault D, Poe D. In vitro and in vivo determination of the thermal effect of middle ear endoscopy. Laryngoscope 1994;106(2):213-216. DOI: 10.1097/00005537-199602000-00020.

6. Kozin ED, Lehmann A, Carter M, et al. Thermal effects of endoscopy in a human temporal bone model: Implications for endoscopic ear surgery. Laryngoscope 2014;124(8):E332-E339. DOI: 10.1002/ lary.24666.

7. Patil RN. Endoscopic tympanoplasty-definitely advantageous (preliminary reports). Asian J Ear Nose Throat 2003;25:913.

8. Ayache S, Tramier B, Strunski V. Otoendoscopy in cholesteatoma surgery of the middleear:what benefits can be expected? Otol Neurotol 2008;29(8):1085-1090. DOI: 10.1097/MAO.0b013e318188e8d7.

9. Presutti L, Marchioni D, Mattioli F, et al. Endoscopic management of acquired cholesteatoma: our experience. J Otolaryngol Head Neck Surg 2008;37(4):481-487.

10. Marchioni D, Mattioli F, Alicandri-Ciufelli M, et al. Endoscopic approach to tensor fold in patients with attic cholesteatoma. Acta Otolaryngol 2009;129(9):946-954. DOI: 10.1080/00016480802468187. 